\title{
CHARACTERISTICS OF DROP IMPACT ON ELASTIC AND COMPLIANT SURFACES
}

Jeng-Horng Chen

Assistant Professor, Department of System and Naval Mechatronic Engineering, National Cheng Kung University Tainan, Taiwan 70101., chenjh@mail.ncku.edu.tw

Follow this and additional works at: https://jmstt.ntou.edu.tw/journal

Part of the Engineering Commons

\section{Recommended Citation}

Chen, Jeng-Horng (2005) "CHARACTERISTICS OF DROP IMPACT ON ELASTIC AND COMPLIANT SURFACES," Journal of Marine Science and Technology. Vol. 13: Iss. 2, Article 11.

DOI: $10.51400 / 2709-6998.2116$

Available at: https://jmstt.ntou.edu.tw/journal/vol13/iss2/11

This Research Article is brought to you for free and open access by Journal of Marine Science and Technology. It has been accepted for inclusion in Journal of Marine Science and Technology by an authorized editor of Journal of Marine Science and Technology. 


\section{CHARACTERISTICS OF DROP IMPACT ON ELASTIC AND COMPLIANT SURFACES}

\section{Acknowledgements}

The author would like to thank Mr. Hao-Ru Fang and Ms. Chu-Ya Huang for their assistance on experiment setup and data sorting. This research was sponsored by National Science Council of the Republic of China (Grant No. NSC 89-2212-E-006-200-). 


\title{
Short Paper
}

\section{CHARACTERISTICS OF DROP IMPACT ON ELASTIC AND COMPLIANT SURFACES}

\author{
Jeng-Horng Chen
}

Key words: drop splash, drop impact.

\section{ABSTRACT}

Due to its importance and wide applications, drop impact on solid and liquid surfaces has been well studied. However, with the progress and wider applications of more sophisticated materials, the relatively less studied physical properties and behavior of a drop impact on elastic or compliant surfaces gradually become more important. Three kinds of common liquid drops impact on both elastic and compliant surfaces were studied experimentally using high-speed digital camera with regular light source at three different impact speeds. The impact effects on compliant surface and the expansioncontraction processes of impacted drops were recorded and analyzed. It is found that Mundo's criterion ( $\mathrm{K}=\mathrm{OhRe}^{1.25}>57.7$ for splashing) based on rigid impact surface experiments is not applicable to elastic and compliant surfaces. However, the maximum spreading diameter is still predictable by $d_{\max } / \mathrm{D}=0.61\left(\mathrm{Re}^{2} \mathrm{Oh}\right)^{0.166}$ which was based on rigid impact surface experiment.

\section{INTRODUCTION}

The phenomenon of liquid drop impact on surfaces is very common in nature and important in many industrial applications, such as spray painting, spray cooling, combustion, ink-jet printers, criminal forensics, fire suppression by sprinkler or water spray, drug spray in biotechnology, and even transportation vehicles in rain. It has been studied since the late $19^{\text {th }}$ century. Most of the studies to date have focused on two aspects: liquid drop impact on rigid and liquid surfaces. Many experimental observations, theories, and even simulations have been reported as seen in [4-8] and references cited therein. In the contrast to these well-studied cases, very little is known about the behavior of a liquid drop impacting on a compliant surface [2] or an elastic surface.

Paper Submitted 10/07/04, Accepted 05/05/05. Author for Correspondence: Jeng-Horng Chen. E-mail: chenjh@mail.ncku.edu.tw.

*Assistant Professor, Department of System and Naval Mechatronic Engineering, National Cheng Kung University Tainan, Taiwan 70101.
These kinds of non-rigid solid surfaces are actually as common as rigid ones in the nature, and also become more and more important in industry and rising biotechnology applications. For example, leaking oil drops falling on high temperature solid surfaces in a machine room will splash, increase oil mist density, and thus raise the risk of fire. A possible solution is to cover hot pipes and machines with materials having compliant surface. More understanding on drop falling on such kind of surfaces could help us verify and develop this fire-prevention method. On medicine applications, drug spray on skins (a kind of compliant surface) is a similar problem. More understanding of drop impact behavior will improve drug spray methods.

The drop impact dynamics is largely controlled by the initial energy state of the fluid that is a function of the mass and velocity $(V)$ of the drop, the drop's original size (diameter, $D$ ), and energy storage and dissipation mechanisms during the impact deformation process, that is density $(\rho)$, surface tension $(\sigma)$ and kinematic viscosity $(v)$. Therefore, these characteristics can be expressed in several dimensionless numbers by dimensional analysis. Reynolds number $(V D / v$, Weber number $\left(\rho V^{2} D / \sigma\right)$, and Ohnesorge number $\left[\mu /(\rho D \sigma)^{1 / 2}=\right.$ $\left(\mathrm{We} / \mathrm{Re}^{2}\right)^{1 / 2}$ ] are usually used. Other parameters such as the interface contact angle and surface roughness also play important roles in impact dynamics, mainly through the determination of the final diameter and possibility of controlling wetting and fingering instabilities along the contact line.

The material behavior of elastic surface may be approached by material mechanics using parameters like surface elasticity and Poisson ratio. On the contrary, the behavior of compliant surfaces was less studied [1, $2]$, and more complex than liquid, rigid or elastic surfaces, mainly due to more structure parameters like damping coefficient, surface thickness, Young's modulus and tension per unit width of the surface [1]. Thus, there is no theoretical approach of drop impingement on these kinds of surfaces to date [3]. The present study's 
objective is to provide qualitative and some quantitative experimental observations for further studies in the future.

\section{EXPERIMENTAL METHOD}

The experiment was designed to generate liquid drops impacting on different kinds of surfaces, including rigid surface, compliant surface, and elastic surface. The drop was generated by carefully and slowly pressing a dropper to let the drop drip off when its weight is larger than surface tension. The drop sizes were examined and appeared to be quite consistent since the size is determined by the liquid surface tension, density and the diameter of dropper's tube. The dropper was set at three different heights to generate different drop impact speeds, and thus different Reynolds numbers. The impact surface was set on the top of a can and was exchangeable. The compliant surface was constructed using a plastic film (BOPP, Bi-axially-Oriented Poly Propylene, $35 \mu \mathrm{m}$ thick, density $=0.91 \mathrm{~g} / \mathrm{cm}^{3}$, produced by Nan-Ya Plastics Co.) with water underneath. The water underneath serves as a damper and may change the property of the impact surface. The elastic surface was constructed in the same way, but with air underneath. Another kind of plastic film (High PHR PVC film for industry use produced by Nan-Ya Plastics Co., tensile strength $=330 \mathrm{~kg} / \mathrm{cm}^{2}, 22 \mu \mathrm{m}$ thick) was also tried for elastic surface for preliminary test of the effect of material on the splashing behavior on elastic surface. The plastic films were fixed on the wall of the can by elastic band without deforming the material during the whole experiment process. Therefore, the surface tensions of the compliant and elastic surfaces were fixed. Other material characteristics of PVC and BOPP films, such as Young's modulus, surface roughness, and tension applied, were neither provided by the manufacturer nor tested in this study. Although these material characteristics are factors affecting the vibration motion of the surfaces, they were fixed in the present study as they are not variables in this experiment. Thus, the limit of the present study is that it can provide qualitative comparison of drop impact behavior on these materials with solid surface. Further studies are needed to understand the effect of material characteristics on the motion of compliant and elastic surfaces after drop impact.

Photo-studio-type continuous light was used for photographic record. A paper diffuser was used in front of the light source. A high speed digital CCD camera (Dalsa CA-D6 with $260 \times 260$ pixel resolution at 955 frame/sec recording rate) was used to record the impact process. The camera was set on the opposite side of light source and took pictures from about 15 degree elevation angle. The images recorded by the camera were sent to a computer immediately for further image processing. The apparatus and instrument configuration is shown in Figure 1. The liquids used in the experiments include water, ethyl alcohol, and lubrication oil $20 \mathrm{~W} / 50$ (China Petroleum Co.). Their properties and drop properties as well as test conditions are listed in Table 1. Low impact velocities were determined from the last two successive images before the drop hit the surface by dividing the distance of the same drop on these two frames by their time interval. For middle and high impact velocities, the velocities were obtained by dividing the streak distance of the drop by camera's exposure time $(0.974 \mathrm{~ms})$ right before impact. The measurement uncertainty caused by these methods was less than $10 \%$.

\section{RESULTS AND DISCUSSION}

\section{Typical impact process}

A drop's typical impact process on a compliant surface or elastic surface is similar to that on a rigid surface. It includes spreading, fingering, and contracting steps. However, there are some differences. Figures 2(a) and 2(b) show a typical water drop impact process on a compliant surface and an elastic surface from $320 \mathrm{~mm}$ height, respectively. It can be seen that the compliant surface formed a waving motion caused by the drop's impact immediately after the impact. A bright circle on the compliant surface was spreading out. The fingering process can also be seen in the picture taken $5.24 \mathrm{~ms}$ after impact. Then, the drop started to contract and capillary waves can be seen inside the outer ring in pictures taken at 13.6, 15.7, and

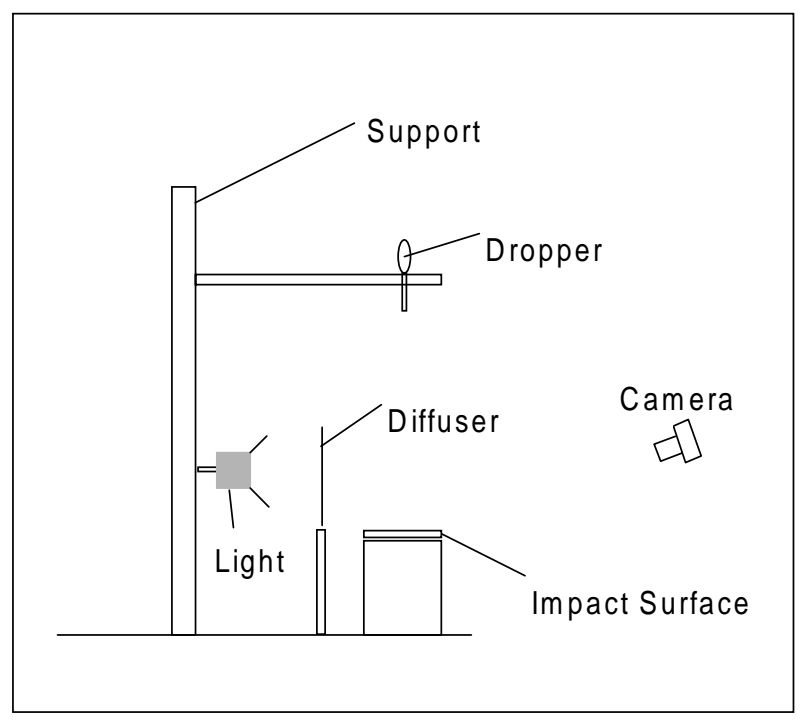

Fig. 1. Experimental setup. 
Table 1. Test conditions*

\begin{tabular}{|c|c|c|c|c|c|c|c|c|c|}
\hline \multirow{2}{*}{$\begin{array}{l}\text { Liquid } \\
\text { Density }\left(\mathrm{Kg} / \mathrm{m}^{3}\right)\end{array}$} & \multicolumn{3}{|c|}{ Water } & \multicolumn{3}{|c|}{ Ethyl alcohol } & \multicolumn{3}{|c|}{ Lubrication oil (20 W/50) } \\
\hline & 998.0 & 998.0 & 998.0 & 789.0 & 789.0 & 789.0 & 887.7 & 887.7 & 887.7 \\
\hline Viscosity $\left(\mathrm{m}^{2} / \mathrm{s}\right)$ & $1.00 \mathrm{E}-06$ & $1.00 \mathrm{E}-06$ & $1.00 \mathrm{E}-06$ & $1.51 \mathrm{E}-06$ & $1.51 \mathrm{E}-06$ & $1.51 \mathrm{E}-06$ & $3.75 \mathrm{E}-04$ & $3.75 \mathrm{E}-04$ & $3.75 \mathrm{E}-04$ \\
\hline Surface tension $(\mathrm{N} / \mathrm{m})$ & $7.28 \mathrm{E}-02$ & $7.28 \mathrm{E}-02$ & $7.28 \mathrm{E}-02$ & $2.28 \mathrm{E}-02$ & $2.28 \mathrm{E}-02$ & $2.28 \mathrm{E}-02$ & $2.60 \mathrm{E}-02$ & $2.60 \mathrm{E}-02$ & $2.60 \mathrm{E}-02$ \\
\hline Drop diameter (mm) & 3.65 & 3.85 & 3.65 & 2.38 & 2.69 & 2.59 & 3.3 & 3.3 & 3.3 \\
\hline Height (mm) & 105 & 320 & 1011 & 105 & 320 & 1011 & 105 & 320 & 1011 \\
\hline Impact velocity $(\mathrm{m} / \mathrm{s})$ & 1.07 & 2.45 & 4.4 & 0.98 & 2.33 & 4.04 & 1.35 & 2.33 & 4.74 \\
\hline $\mathrm{Re}$ & 3906 & 9433 & 16060 & 1545 & 4151 & 6930 & 11.9 & 20.5 & 41.7 \\
\hline We & 57 & 317 & 969 & 79 & 505 & 1463 & 205 & 612 & 2531 \\
\hline Oh $(\times 1000)$ & 1.94 & 1.89 & 1.94 & 5.76 & 5.42 & 5.52 & 1206 & 1206 & 1206 \\
\hline \multicolumn{10}{|l|}{ Max. spreading diameter } \\
\hline Compliant surface (mm) & 12 & 15.8 & 21.3 & 9.8 & 11.6 & 12.7 & 5.6 & 5.7 & 7.3 \\
\hline Elastic surface $(\mathrm{mm})$ & 12.2 & 17.2 & 20.2 & 10.2 & 12.2 & 13.3 & 5.1 & 5.5 & 7.1 \\
\hline $\mathrm{K}=\mathrm{OhRe}^{1.25}$ & 60 & 175 & 350 & 56 & 180 & 349 & 27 & 53 & 128 \\
\hline Splashing on compliant surface? & No & No & $?$ & No & No & No & No & No & No \\
\hline Splashing on BOPP surface? & No & No & No & No & No & No & No & No & No \\
\hline Splashing on PVC surface? & No & No & Yes & No & No & No & No & No & No \\
\hline
\end{tabular}

*Experiments were conducted at $20^{\circ} \mathrm{C}$.
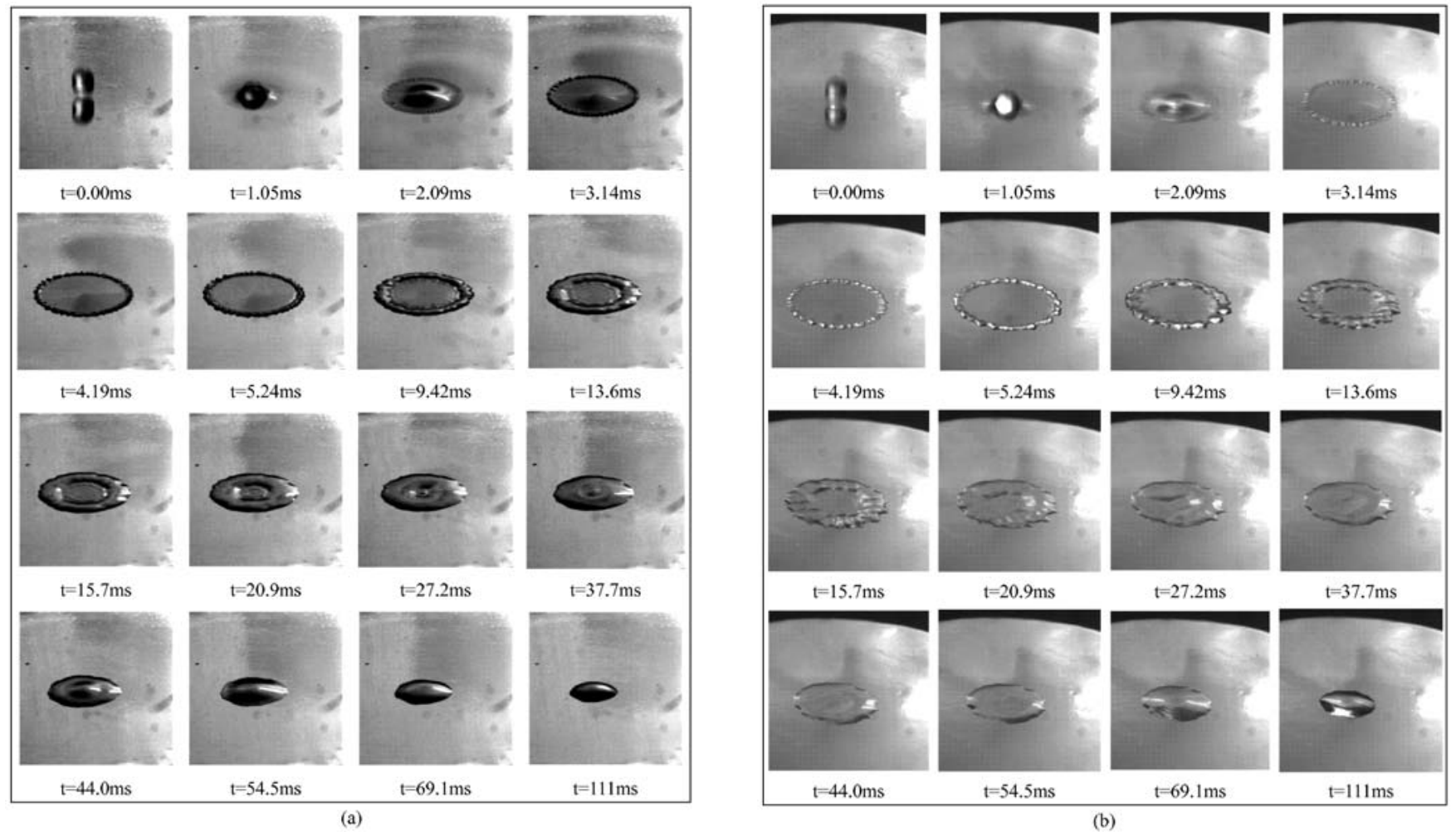

Fig. 2. (a) Typical water drop impact process on a compliant surface (dropping height $=320 \mathrm{~mm}$ ). (b) Typical water drop impact process on an elastic surface (dropping height $=320 \mathrm{~mm}$ ).

$20.9 \mathrm{~ms}$ after impact. Finally, the drop contracted to a half sphere shape. No splashing at all was observed. The waving motion of elastic surface after drop impact was less obvious than that of compliant surface, mainly due to its much faster vibration speed and small amplitude observed from the change of light reflection area 
on its surface.

\section{Maximum spreading diameter}

Early findings of various aspects of liquid drop impact on rigid and liquid surfaces were reviewed by Rein [6]. Usually, the maximum spreading diameter, dmax, is correlated with Reynolds number and Weber number. But, Scheller and Bousfield recently proposed a more accurate empirical formula to correlate nondimensional maximum spreading diameter, $d_{\max } / D$, with Reynolds number and Ohnesorge number for rigid impact surface [7]:

$$
d_{\max } / D=0.61\left(\operatorname{Re}^{2} \mathrm{Oh}\right)^{0.166}
$$

Due to the lack of appropriate theory of drop impact on compliant and elastic surfaces, the present experiment results of non-dimensional maximum spreading diameter was shown in Figure 3 with Equation (1). Although the present data are for elastic or compliant impact surfaces, the correlation seems to work as well. This may be explained by the fact that the elastic and compliant surfaces used in the experiments are still material in solid phase, thus, having material characteristics closer to rigid surface than to other kinds of interfaces (such as liquid). Hence, the softer material characteristics may play roles on affecting energy distribution and transition during impact process, but not affecting the basic mode of impact. The present observation implies that a theoretical about the factors affecting the maximum diameter is needed to justify the use of equation (1) on compliant and elastic surfaces.

\section{Splashing}

Whether a drop impact event will lead to liquid

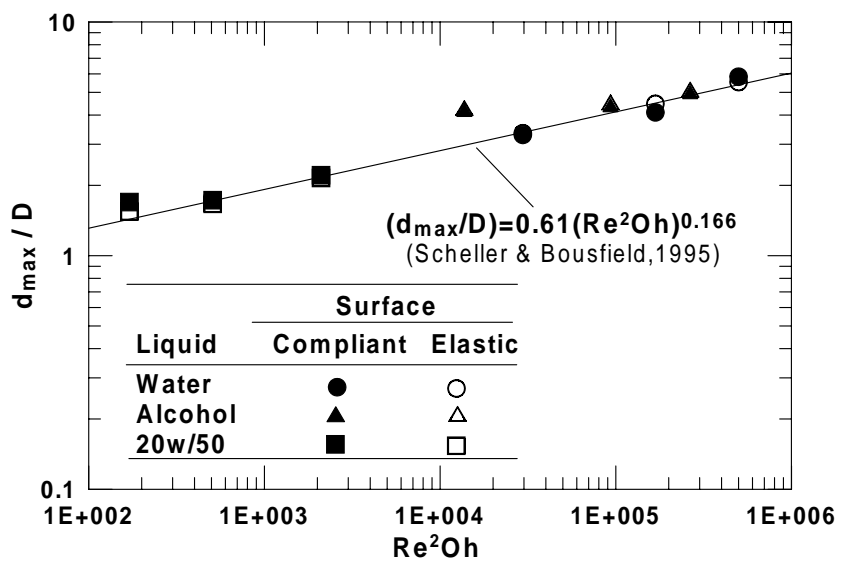

Fig. 3. Non-dimensional maximum spreading diameters as a function of $\left(\operatorname{Re}^{2} \mathrm{Oh}\right)$. splashing is very important to many applications, such as in the spray painting or ink-jet printer cases. Thus, splashing has been another focus in the study of drop impact. Various kinds of methods to reduce or prevent splashing are under investigating, including changing fluid properties and changing impact surface properties. Most past studies concentrated on drop impact on rigid surfaces. Mundo proposed a criterion of splashing condition for rigid impact surface based on experiments [5]:

$$
K=\mathrm{OhRe}^{1.25}>57.7
$$

If $K$ is larger than a critical empirical value 57.7, then it is in splashing regime. However, this empirical formula does not include other factors such as surface roughness, which is known to affect the contact angle between liquid drop and solid surface. Thoroddsen and Sakakibara's experiments on rigid impact surface [8] showed that some of their test conditions in Mundo's splashing regime but close to the splashing line did not splash. They suspected that surface roughness plays an important role and caused the ineffectiveness of Mundo's criteria, i.e., rougher solid surface will trigger splashing for drops in conditions near the splashing line. Hence, the critical value of $K$ might need correction for roughness. Thus, it can be considered as a useful approximation.

Nevertheless, in lack of appropriate theory of drop impact on compliant and elastic surfaces, the present results of drop impact on compliant surface and elastic surface were compared with Mundo's formula and shown in Figure 4. It is clear that six of the present results are in the splashing regime. But splashing behavior was

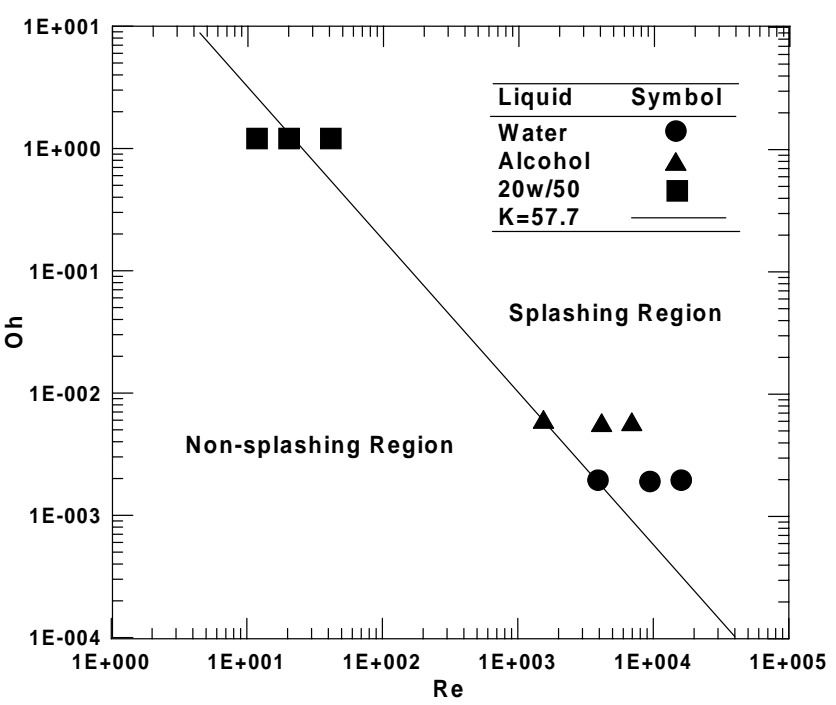

Fig. 4. Splashing regime. 


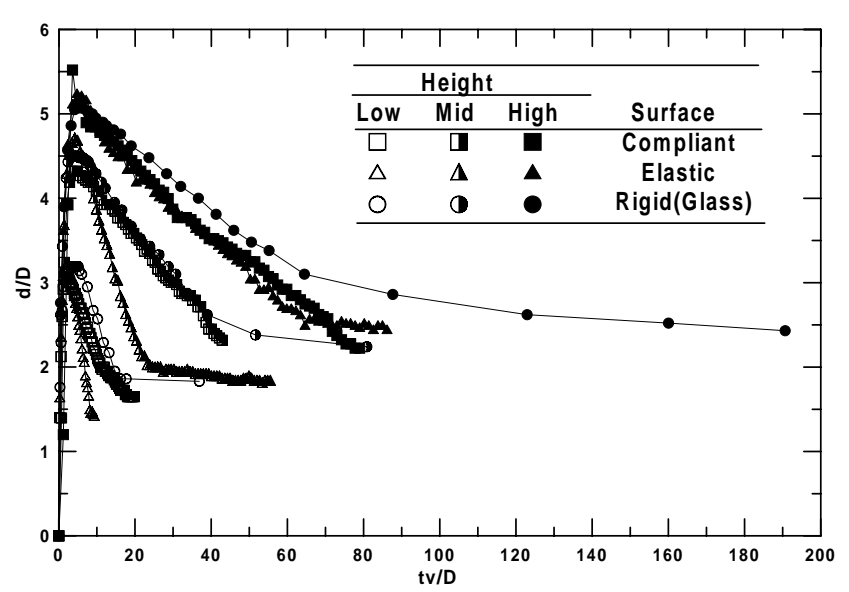

Fig. 5. Water drop spreading process.

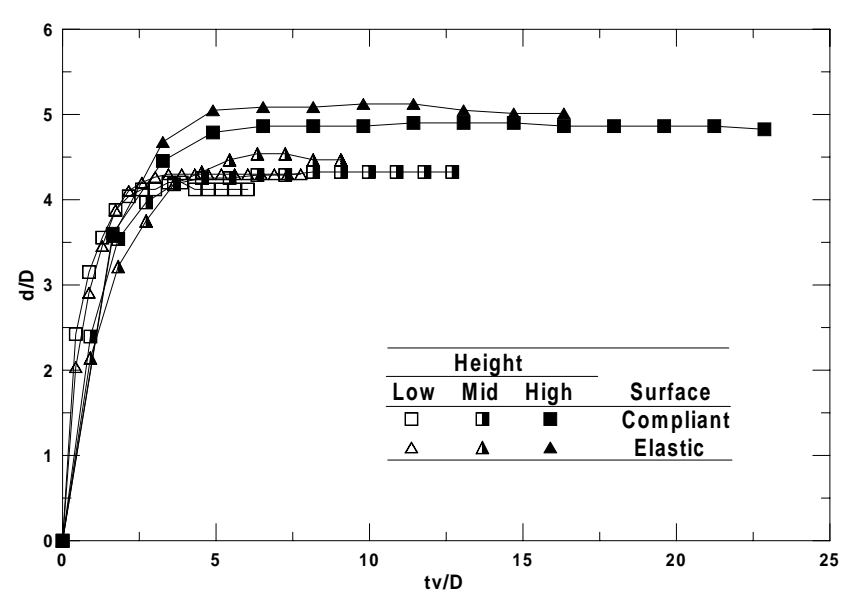

Fig. 6. Ethyl alcohol drop spreading process.

almost not observed for all compliant surface cases. Only the water drops with largest Re impacting on compliant surface showed splashing in only half time of all tests. The question mark in Table 1 denotes this observation. The $K$ values of these six conditions are either larger or much larger than Mundo's critical value suggesting that roughness is not a critical factor affecting splashing phenomenon in this study. Thus, we need other reasons to explain its deviation from splashing. Since the waving motion of compliant surface can be clearly seen in the pictures taken, we suspect two mechanisms decreases the energy for splashing: the energy given to the compliant surface to produce waving motion, and the energy absorbed by water underneath.

In the present study, splashing did occur in part of the elastic impact surface cases. Splashing was not observed on the BOPP elastic surface in all conditions.

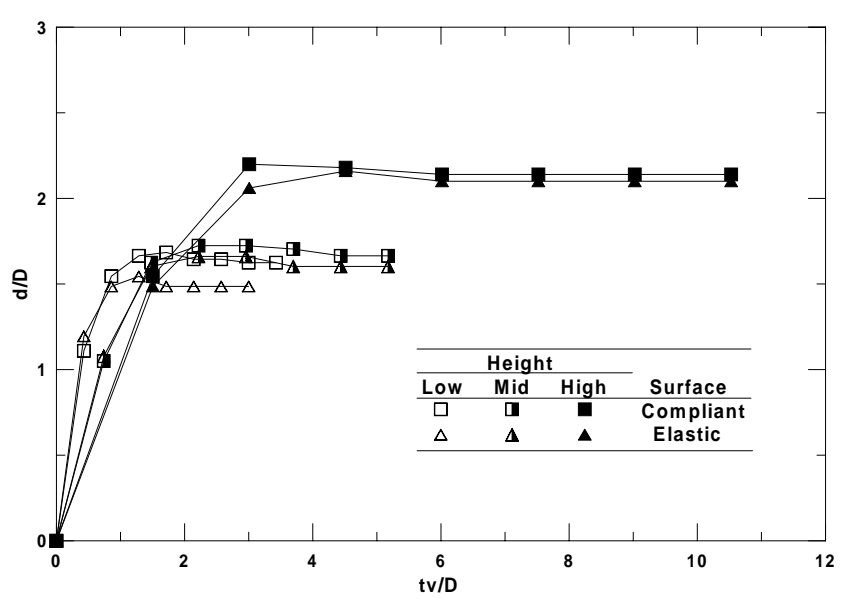

Fig. 7. Lubrication oil drop spreading process.

However, splashing occurred only in the largest Reynolds number case of water drops impacting on PVC elastic surface. By comparing with the same conditions on compliant surface, it is suspected that the bouncing phenomenon or the different waving motion of the surface "help" the drop to splash during the spreading process. Thus, the material characteristics of impact surfaces have an effect on whether the splashing occurs after impact. The PVC elastic surface seems to make splashing easier than BOPP surface.

\section{Evolution of spreading process}

Figures 5, 6, and 7 show the evolution of spreading diameter of water, alcohol, and lubrication oil drops after impact on compliant surface and BOPP elastic surface. The spreading diameter, $d$, is normalized by its original diameter, $D$, and the time, $t$, is normalized by impacting velocity, $V$, and drop's original diameter. For water drops, we also compare the present results of impacting on elastic and compliant surfaces with those on rigid (glass) impact surface. The spreading processes of water drop on three kinds of surfaces are shown in Figure 5. The processes are similar for the high impact velocity case, except that drop seemed to contract slightly quicker on both elastic and compliant surfaces than on rigid surface.

However, in the other two cases, only the drop on elastic surface contracted obviously faster than on the other two surfaces. The spreading process of ethyl alcohol is shown in Figure 6. It is clear that alcohol drops almost did not contract after expanding to their maximum diameters on both kinds of surfaces. Similar results for lubrication oil drops can be seen on Figure 7. However, lubrication oil drops demonstrate different behavior regarding the impact surface effect. They 
reached slightly larger diameters on compliant surface than on elastic surface. This is exactly the opposite of ethyl alcohol drops.

Comparing the spreading processes of these three kinds of liquid drops, we found that lubrication oil has the smallest normalized maximum spreading diameter, as seen in Figure 3. However, since it doesn't contract much after spreading, the final normalized diameters are close to water drops' normalized diameter after water drops contract. On the other hand, ethyl alcohol's final normalized diameters are the largest. It is also clear that ethyl alcohol and lubrication oil reach their final diameter much faster than water at the same test condition. Water drops usually spend much time in the contracting process. Contrarily, both alcohol and oil set to the final diameter faster because they do not have any obvious contracting process.

\section{CONCLUSION}

Liquid drop impacting on both compliant and elastic surfaces have been less studied, but might have useful applications in the future. The present study found the following characteristics:

1. The maximum spreading diameter of drop impact on compliant surface and elastic surface is still predictable by $d_{\max } / D=0.61\left(\operatorname{Re}^{2} \mathrm{Oh}\right)^{0.166}$ which was based on rigid surface experiment.

2. Splashing after liquid drop impact on elastic or compliant surfaces is a complicated problem, and thus Mundo's criterion ( $K=\mathrm{OhRe}^{1.25}>57.7$ for splashing) based on rigid surface experiments is not applicable to elastic and compliant surfaces.

3 . The surface property (compliant or elastic) and material property both have effects on the drop-surface interaction and thus affect splashing condition. A further theoretical approach on this matter is needed.

4. Lubrication oil drops and ethyl alcohol drops almost do not contract after they reach their maximum spreading diameters.

5. The contracting processes of water drops on both compliant surface and elastic surface are similar to those on rigid surface, except that they contract more quickly on either compliant surface or elastic surface.

6 . The contracting process of drop impact on compliant or elastic surfaces needs more experimental and theoretical studies in the future.

\section{ACKNOWLEDGEMENTS}

The author would like to thank Mr. Hao-Ru Fang and Ms. Chu-Ya Huang for their assistance on experiment setup and data sorting. This research was sponsored by National Science Council of the Republic of China (Grant No. NSC 89-2212-E-006-200-).

\section{REFERENCES}

1. Carpenter, P.W. and Garrad, A.D., "The Hydrodynamic Stability of Flow over Kramer-type Compliant Surfaces Part 1. Tollmien-Schlichting Instabilities,' J. Fluid Mech., Vol. 155, pp. 465-510 (1985).

2. Field, J.E., Dear, J.P., and Ogren, J.E., "The Effects of Target Compliant Surface on Liquid Drop Impact," $J$. Appl. Phys., Vol. 65, No. 2, pp. 533-540 (1988).

3. Kiger, K. and Chen, J.-H., Characteristics of Drop Impingement on Compliant Surfaces, Report to Bowles Fluidics Corporation (2000).

4. Manzello, S.L. and Yang, J.C., "An Experimental Study of a Water Droplet Impinging on a Liquid Surface," Exp. Fluid, Vol. 32, pp. 580-589 (2002).

5. Mundo, C.H.R., Sommerfeld, M., and Tropea, C., "Droplet-Wall Collision: Experimental Studies of the Deformation and Breakup Process," Int. J. Multiphas. Flow, Vol. 21, No. 2, pp. 151-173 (1995).

6. Rein, M., "Phenomena of Liquid Drop Impact on Solid and Liquid Surfaces," Fluid Dyn. Res., Vol. 12, pp. 6193 (1993).

7. Scheller, B.L. and Bousfild, D.W., "Newtonian Drop Impact with a Solid Surface," AIChE J., Vol. 41, No. 6, pp. 1357-1367 (1995).

8. Thoroddsen, S.T. and Sakakibara, J., "Evolution of the Figering Pattern of an Impacting Drop," Phys. Fluids, Vol. 10, No. 6, pp. 1359-1374 (1998). 